\title{
Novel canine isocitrate dehydrogenase 1 mutation Y208C attenuates dimerization ability
}

\author{
SHOTA KAWAKAMI ${ }^{1}$, MASAKI MICHISHITA ${ }^{2,3}$, MOTOHARU SAKAUE $^{4}$, MASAMI MORIMATSU $^{5}$, \\ MITSUKI UEMURA $^{1,6}$, NOBUAKI KASHIWAGI ${ }^{1,6}$, MARIKA MAEDA ${ }^{6}$, YUKINO MACHIDA ${ }^{2}$, \\ DAIGO AZAKAMI $^{7}$, AI S. EGUSA ${ }^{8}$, ERI ONOZAWA ${ }^{1}$, KATSUMI ISHIOKA $^{1}$, MASAMI WATANABE $^{9}$, \\ YOSHIKAZU TANAKA ${ }^{3,6}$, TOSHINORI OMI ${ }^{1,3}$ and KAZUHIKO OCHIAI ${ }^{1,3,6}$
}

\begin{abstract}
${ }^{1}$ School of Veterinary Nursing and Technology, Faculty of Veterinary Science; ${ }^{2}$ Laboratory of Veterinary Pathology, School of Veterinary Science; ${ }^{3}$ Research Center for Animal Life Science, Nippon Veterinary and Life Science University, Musashino, Tokyo 180-8602; ${ }^{4}$ Laboratory of Anatomy II, Department of Veterinary Medicine, School of Veterinary Medicine, Azabu University, Sagamihara, Kanagawa 252-5201; ${ }^{5}$ Laboratory of Animal Science and Medicine, Department of Disease Control, Graduate School of Veterinary Medicine, Hokkaido University, Sapporo 060-0818; ${ }^{6}$ Laboratory of Veterinary Hygiene, School of Veterinary Science, Nippon Veterinary and Life Science University, Musashino, Tokyo 180-8602; ${ }^{7}$ Laboratory of Clinical Oncology, Cooperative Department of Veterinary Medicine,

Faculty of Agriculture, Tokyo University of Agriculture and Technology, Fuchu, Tokyo 183-8538;

${ }^{8}$ Department of Applied Life Science, Faculty of Food Science, Nippon Veterinary and Life Science University, Musashino, Tokyo 180-8602; ${ }^{9}$ Department of Urology, Graduate School of Medicine, Dentistry and Pharmaceutical Sciences, Okayama University, Okayama 700-8558, Japan
\end{abstract}

Received June 12, 2020; Accepted September 11, 2020

DOI: $10.3892 / 01.2020 .12214$

\begin{abstract}
Isocitrate dehydrogenase 1 (IDH1) mutations are common in gliomas, acute myeloid leukemia, and chondrosarcoma. The mutation 'hotspot' is a single arginine residue, $\mathrm{R} 132$. The R132H mutant of IDH1 produces the 2-hydroxyglutarate (2-HG) carcinogen from $\alpha$-ketoglutarate $(\alpha-K G)$. The reduction of $\alpha-K G$ induces the accumulation of hypoxia-inducible factor- $1 \alpha$ subunit (HIF-1 $\alpha$ ) in the cytosol, which is a predisposing factor for carcinogenesis. $\mathrm{R} 132 \mathrm{H}$ is the most common IDH1 mutation in humans, but mutations at the R132 residue can also occur in tumor tissues of dogs. The current study reported the discovery of a novel Tyr208Cys (Y208C) mutation in canine IDH1 (cIDH1), which was isolated from 2 of 45 canine chondrosarcoma cases. As the genomic DNA isolated from chondrosarcoma tissue was mutated, but that isolated from blood was not, Y208C mutations were considered to be spontaneous somatic mutations. The isocitrate dehydrogenase activity of the Y208C mutant was attenuated compared
\end{abstract}

Correspondence to: Dr Kazuhiko Ochiai, Laboratory of Veterinary Hygiene, School of Veterinary Science, Nippon Veterinary and Life Science University, 1-7-1 Kyonan-cho Musashino, Tokyo 180-8602, Japan

E-mail: kochiai@nvlu.ac.jp

Key words: canine, chondrosarcoma, dimerization, isocitrate dehydrogenase 1 , glioma, mutation with that of wild-type (WT) cIDH1, but the attenuation of Y208C was less intense than that of the R132H mutation. The induction of HIF- $1 \alpha$ response element activity and cell retention of HIF-1 $\alpha$ were not increased by Y208C overexpression. In silico and cell biological analysis of IDH1 dimerization revealed that the Y208C mutation, but not the R132H mutation, attenuated binding activity with WT cIDH1. These data suggested that the attenuation of dimerization by the Y208C mutation may cause tumorigenesis through different mechanisms other than via 2-HG production by the IDH1 R132 mutation.

\section{Introduction}

Gliomas are intracranial tumors that are thought to develop from astrocytes or oligodendrocytes $(1,2)$. A genome-wide mutation analysis of human brain tumors revealed predominant somatic mutations in the gene encoding isocitrate dehydrogenase 1 (IDH1) in glioblastoma (GBM) $(3,4)$. Further analysis of the IDH1 gene structure confirmed these findings, identifying IDH1 mutations in over $70 \%$ of secondary GBM or low-grade gliomas, but infrequently in primary $\operatorname{GBM}(5,6)$. Almost all IDH1 mutations were identified at the 132th arginine residue (R132). In addition, R132 mutations of IDH1 have been identified in acute myeloid leukemia (AML) and chondrosarcoma (CS) (7-9). R132 mutants of IDH1 induced the reduction of $\alpha$-ketoglutarate $(\alpha-\mathrm{KG})$ and increased the 2-hydroxyglutarate (2-HG) production, whereas converting nicotinamide adenine dinucleotide phosphate (NADPH) to $\mathrm{NADP}^{+}(10-12)$. The $2-\mathrm{HG}$ increase in the brain propagates 
reactive oxygen species, leading to a variety of after-effects (13). A decrease in $\alpha-K G$ with an increase in 2-HG causes the reduction of $\alpha$-KG-dependent prolyl hydroxylases, such as those that regulate hypoxia-inducible factor- $1 \alpha$ subunit (HIF-1 $\alpha$ ) levels. Alterations in HIF-1 $\alpha$ have been reported to result from mutant IDH1 protein expression (14), causing oncogenic transformation. In addition, the loss or gain of IDH1 function without R132 mutation is also related to cancer progression and resistance to chemotherapy as mediated by NADPH biosynthesis (15-17). Therefore, it is important to focus on mutations other than the $\mathrm{R} 132 \mathrm{H}$ mutation, in order to further investigate IDH1 molecular function and cancer development.

Although intracranial tumors in dogs, such as meningiomas and gliomas, are relatively common brain diseases (18), partial sequencing analysis targeting R132 in IDH1 has been performed, but no mutations have been identified in canine gliomas (19). We recently cloned full-length cDNA of cIDH1 and performed artificial R132 mutation analysis of cIDH1 (20). The antibodies used to detect the specific R132 mutation in humans could also be used to detect R132 mutations in cIDH1 $(21,22)$, and the production ability of NADPH was attenuated by the R132 mutation of cIDH1. Furthermore, the R132H mutant of cIDH1 intensified HIF-1 $\alpha$ expression, showing that the R132 mutation in cIDH1 plays a potential role in tumor predisposition. The most recent study to show that the R132C mutation was found in canine glioma cases (23) did not found any other mutations in cIDH1.

In this study, we found the novel mutation Y208C in cIDH1 by sequencing formalin-fixed paraffin-embedded (FFPE) canine chondrosarcoma tissues. We compared the production ability of NADPH and induction of HIF-1 $\alpha$ between the wild-type (WT) and cIDH1 mutants. Furthermore, the dimerization ability necessary to exert the enzyme activity of IDH1 was estimated in silico and measured by cell biology analysis.

\section{Materials and methods}

Sample preparation and sequencing. The genomic DNA of the FFPE tissue from paraffin scrolls (Table SI) was extracted from canine tumor samples using the QIAamp DNA FFPE Tissue Kit (Qiagen) according to the manufacturer's instructions. PCR amplification was performed using PrimeSTAR (Takara). Primer pairs used for amplifying cIDH1 exons are listed in Table I. Sequence data were directly determined using an ABI 3100-Avant Genetic Analyzer (Applied Biosystems). For the sequence analysis, human IDH1 (GenBank accession no. NP_005887.2) and cIDH1 (BBC43078.1) were compared accordingly (Fig. 1A).

Histological analysis. With permission from the University Ethics Committee, we obtained tissue samples from the Department of Veterinary Pathology, School of Veterinary Science, Nippon Veterinary and Life Science University (approval no. 11-50, 27 May 2018). All samples were classified by veterinary pathologists according to the World Health Organization classification (24) (Table SI). FFPE cancer tissues were sliced at a thickness of $4 \mu \mathrm{m}$, the sections were placed on slides, and hematoxylin and eosin (H\&E) staining was performed.
Cell cultures. HeLa and MDCK cells were purchased from the American Type Culture Collection (ATCC). The cell lines were maintained in Dulbecco's modified Eagle's medium (Wako) supplemented with 10\% fetal bovine serum, penicillin, and streptomycin (Applied Biosystems) and incubated at $37^{\circ} \mathrm{C}$ in a $5 \% \mathrm{CO}_{2}$ atmosphere.

Measurement of the isocitrate dehydrogenase activity. To measure the production of NADH and NADPH, cIDH1-transfected cells $\left(5 \times 10^{4}\right)$ were processed using the Isocitrate Dehydrogenase Activity Colorimetric Assay Kit (BioVision) according to the manufacturer's instructions. The reaction mix was treated for $10 \mathrm{~min}$, and the optical density at $450 \mathrm{~nm}$ was measured using an iMark microplate reader (Bio-Rad Laboratories).

Measurement of the HIF-1 $\alpha$ promoter activity. HeLa cell transfection was performed in a 96-well plate at $80 \%$ confluency. The vector containing the HIF-1 $\alpha$ response element pGL4.42[luc2P/HRE/Hygro] (Promega) was co-transfected with the control vector phRL-TK (Promega) as a transfection efficiency control. Forty-eight hours later, luciferase activity was measured using the Dual-Glo Luciferase Assay System (Promega). Luciferase activity was normalized to that of Renilla luciferase activity.

$\alpha-K G$ assays. HeLa cells were harvested in a 24-well plate at a density of $1 \times 10^{5}$ cells/well and transfected with $250 \mathrm{ng}$ of HA-tagged, full-length WT, R132H, or Y208C mutant of cIDH1 in pMACS Kk.HA-C (Miltenyi Biotec). The assay was performed using the coupled enzymatic assay method according to the manufacturer's instructions (Sigma-Aldrich; Merck KGaA, catalog no. MAK054). In this method, $\alpha-\mathrm{KG}$ concentration is determined by a coupled enzyme assay, which results in a colorimetric $(570 \mathrm{~nm})$ product that, in turn, is proportional to the amount of $\alpha-K G$ present in the sample.

Induction of HIF-1 $\alpha$ expression by $\mathrm{CoCl}_{2}$. For the $\mathrm{CoCl}_{2}$ (Wako) experiments to induce HIF-1 $\alpha$ expression, $2 \times 10^{5} \mathrm{HeLa}$ cells were seeded in 6-well plates for $24 \mathrm{~h}$ before being treated with $100 \mu \mathrm{M} \mathrm{CoCl}_{2}$ for an additional $24 \mathrm{~h}$.

Immunoblotting. Immunoblotting was performed using the following primary antibodies: Rabbit polyclonal anti-HA (561, 1:1,000; MBL), anti- $\beta$-actin (PM053, 1:2,000; MBL), rabbit polyclonal anti-HIF-1 $\alpha$ (\#3716, 1:1,000; Cell Signaling Technology), and anti-Halo antibody (G9281, 1:1000; Promega). Horseradish peroxidase-conjugated secondary antibodies and EzWestLumi plus (ATTO) were used for detecting antibody-bound proteins.

Crystal structure modeling. We retrieved the crystal structure of the human IDH1 dimer from the Research Collaboratory for Structural Bioinformatics Protein Data Bank at http://www.rcsb.org/ (PDB ID: 5YFM) and analyzed it using the University of California, San Francisco Chimera software (http://www.cgl.ucsf.edu/chimera/) (25).

Mammalian cell two-hybrid assay. For the mammalian cell two-hybrid assay (MTH), WT and Y208C cIDH1 cDNA 
Table I. Primer pairs to amplify canine Isocitrate dehydrogenase 1 exons.

\begin{tabular}{lll}
\hline Exon & \multicolumn{1}{c}{ Primer sequences } \\
\hline 3 & F: & 5'-GCAGCCTCAAAAGCCACACACGC-3' \\
& R: & 5'-TGTACTTATCTTTAAGCATCCC-3' \\
4 & F: 5'-CGTTGTGCGCCATCACACAG-3' \\
& R: 5'-CACTTAAAGGGAGTAGTCAC-3' \\
5 & F: 5'-TGATCTTGAGTCTATACCAG-3' \\
& R: 5'-TGGCTAGTTCCCTTTGTGTC-3' \\
6 & F: 5'-GACTTTCTTCCAATCACGTG-3 \\
& R: 5'-TATGCCCTTAACTTTATGGG-3' \\
7 & F: 5'-GCCTGATGCAAGACTCGATC-3' \\
& R: 5'-TTCATTGATGACTACACATGC-3' \\
8 & F: 5'-GGACCCTGCTTCCTGAGAGG-3' \\
& R: 5'-GGACCCTGCTTCCTGAGAGG-3' \\
9 & F: 5'-TCTGCTCAACAGCAAGACAG-3' \\
& R: 5'-TGACTGTGCTCCTTCCACAG-3' \\
10 & F: 5'-GTGGCCGAGCTGCCAGTGCAGGC-3' \\
& R: 5'-CCTGCCACGTTCACGAGGGTG-3'
\end{tabular}

F, forward; R, reverse.

were cloned into the EcoRI and MluI sites of the pM GAL4 DNA-binding domain of the GAL4-DBD plasmid (pM) (Clontech Laboratories) and the pVP16 transactivation domain of the VP16-AD plasmid (pVP16) (Clontech Laboratories), respectively. Approximately $2 \times 10^{5} \mathrm{HeLa}$ cells were placed in a 24-well plate and were co-transfected with $100 \mathrm{ng}$ of $\mathrm{pM}$, $100 \mathrm{ng}$ of pVP16, $100 \mathrm{ng}$ of pFR-Luc firefly luciferase reporter plasmid (Promega), and 2 ng of phRL-TK Renilla luciferase reporter plasmid (Promega). The cells were harvested $48 \mathrm{~h}$ after transfection, and luciferase activity was measured using a Dual-Glo Luciferase Assay System (Promega). Luciferase activity was normalized to that of Renilla luciferase activity.

Pull-down assay. We cloned the Halo- or HA-tagged, full-length WT, R132H or Y208C mutant into the pFN21A (Promega) or pMACS Kk.HA-C vector, respectively. The expression of the Halo- and HA-tagged constructs in the HeLa cells was induced using FuGENE HD Transfection Reagent (Promega), and the transfected cells were grown for $48 \mathrm{~h}$. The cells were then lysed and pulled down using Mammalian Lysis Buffer (Promega) containing Protease Inhibitor Cocktail (Promega) for $15 \mathrm{~min}$, and cellular debris was cleared by centrifugation at $12,000 \mathrm{x}$ g for $10 \mathrm{~min}$. In total, $50 \mu 1$ of Magne Halo-Tag Beads (Promega) equilibrated with TBS containing $0.05 \%$ IGEPAL CA-630 (TBS+) was added to the supernatant. The samples were incubated for $20 \mathrm{~min}$ at $22^{\circ} \mathrm{C}$ with rotation. The supernatant was discarded, and the protein-captured beads were washed thrice with $\mathrm{TBS}+$ and suspended in SDS-PAGE loading buffer. The samples were analyzed by immunoblotting using anti-Halo or anti-HA antibody and horseradish peroxidase-conjugated anti-rabbit IgG antibody (GE Healthcare). The blots were developed using EzWestLumi plus reagents.
Sample preparation, cross-linking procedure and detection of cIDH1 dimer. HeLa cells in 6-well plates were transfected with either WT, R132H or Y208C of cIDH1 expression plasmids (1 $\mu \mathrm{g} /$ well). After $48 \mathrm{~h}$ of transfection, the cells were lysed with mammalian lysis buffer (Promega) supplemented with a protease inhibitor cocktail (Promega). Post lysis, the samples were centrifuged $\left(15,000 \mathrm{x} \mathrm{g}\right.$ for $15 \mathrm{~min}$ at $\left.4^{\circ} \mathrm{C}\right)$ to obtain the supernatant accordingly. Total protein levels were measured using BCA (Nacalai Tesque). Equal amounts of proteins (100 $\mu \mathrm{g} /$ condition) were then incubated with glutaraldehyde at different concentrations $(0,0.04,0.1,0.25$ or $0.5 \%)$ and incubated on ice for $30 \mathrm{~min}$ accordingly. To make a working solution of glutaraldehyde, commercially available $25 \%$ glutaraldehyde solution was diluted in PBS and discarded after use. To quench the reaction, sample buffer was added to obtain the following final concentrations: $250 \mathrm{mM}$ Tris- $\mathrm{HCl}, \mathrm{pH} 8.5 ; 2 \%$ lithium dodecyl sulfate; 100 mM DTT; 0.4 mM EDTA; 10\% glycerol; and $0.2 \mathrm{mM}$ bromophenol blue. Samples were then separated by SDS-PAGE and the monomer and dimer of cIDH1 was detected using anti-HA antibody accordingly.

NADPH oxidase (NOX) activity assay. NOX activity was evaluated by assessing the superoxide production by lucigenin-enhanced chemiluminescence (26). WT or mutant cIDH1-transfected HeLa cells were disrupted in $0.2 \mathrm{ml}$ of extraction buffer (20 mM sodium phosphate buffer ( $\mathrm{pH} 7.0$ ), $1 \mathrm{mM}$ EDTA, $0.5 \mathrm{mM}$ PMSF, $1 \mu \mathrm{g} / \mathrm{ml}$ Aprotinin, $0.5 \mu \mathrm{g} / \mathrm{ml}$ leupeptin) using a homogenizer on ice. After the homogenates were centrifuged at $1,000 \mathrm{xg}$ for $10 \mathrm{~min}$ at $4^{\circ} \mathrm{C}$, the supernatant $(20 \mu \mathrm{l})$ was added to $0.2 \mathrm{ml}$ of assay buffer $(50 \mathrm{mM}$ sodium phosphate buffer ( $\mathrm{pH} 7.0$ ), $1 \mathrm{mM}$ EDTA, $150 \mathrm{mM}$ sucrose, and $50 \mathrm{mM}$ lucigenin). After the addition of $0.1 \mathrm{mM} \mathrm{NADPH}$, luminescence was measured as relative light units (RLUs) at 15-sec intervals for 1,000 msec in a luminometer (GLOMAX; Promega). NOX activity was indicated as RLUs per minute per $\mathrm{mg}$ of protein.

Statistical analysis. Data are expressed as mean \pm standard deviation (SD). Analysis of variance (ANOVA) with a Tukey's post-hoc test was used when multiple comparisons were required. $\mathrm{P}<0.01$ was considered statistically significant.

\section{Results}

Isolation of the Y208C mutation from canine CS. Genomic DNA was isolated from tumor tissues and blood. PCR amplification of the coding exons of cIDH1 (Fig. 1B) and sequence analysis of IDH1 in 45 tumor samples (Table SI) revealed two genetic alternations in CS, from tyrosine to cysteine at residue 208 (Y208C). No other somatic mutations in cIDH1 were found in these samples. These two case's genome DNA, isolated from blood, were not found to be mutated (Fig. 1C). Fig. 1D shows representative CS samples with Y208C (case no. 7 in Table SI) H\&E-stained. The proliferation of neoplastic chondrocytes with abundant chondroid matrix was observed.

Attenuation of NADPH and $\alpha-K G$ production in Y208C-mutant IDH1 cells. The formation of NADPH in multiple types of cIDH1-overexpressing HeLa and MDCK cells was measured using colorimetric analysis. The productivity of NADPH in the 
A

human IDH1 1 MSKKISGGSVVEMQGDEMTRI IWELIKEKLIFPYVELDLHSYDLGIENRDATNDQVTKDA 60

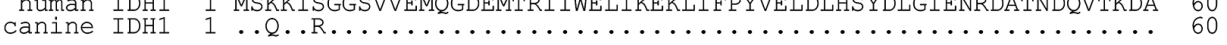

61 AEAIKKHNVGVKCATITPDEKRVEEFKLKQMWKSPNGTIRNILGGTVFREAIICKNIPRL 120

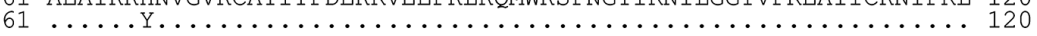

121 VSGWVKPIIIGRHAYGDQYRATDFVVPGPGKVEITYTPSDGTQKVTYLVHNEEEGGGVAM 180

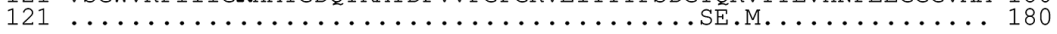

181 GMYNQDKSIEDFAHSSFQMALSKGWPLYLSTKNTILKKYDGRFKDIFQEIYDKQYKSQFE 240

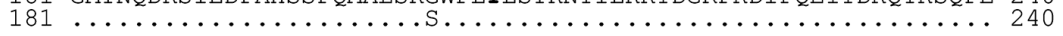

241 AQKIWYEHRLIDDMVAQAMKSEGGFIWACKNYDGDVQSDSVAQGYGSLGMMTSVLVCPDG 300

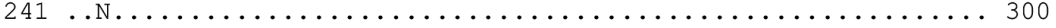

301 KTVEAEAAHGTVTRHYRMYQKGQETSTNPIASIFAWTRGLAHRAKLDNNKELAFFANALE 360

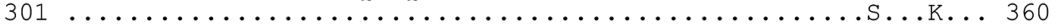

361 EVSIETIEAGFMTKDLAACIKGLPNVQRSDYLNTFEFMDKLGENLKIKLAQAKL 414

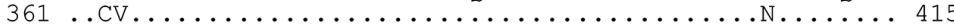

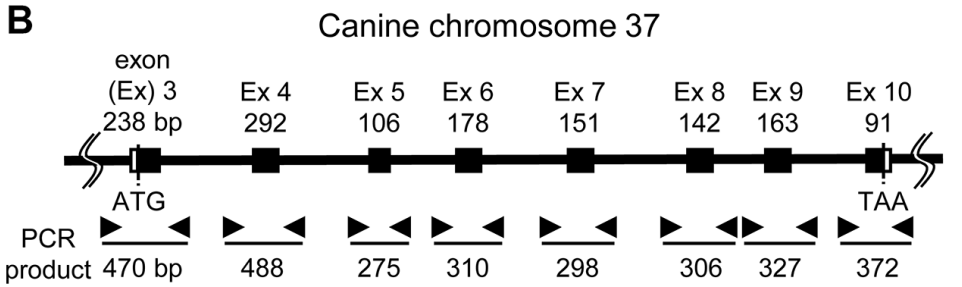

C

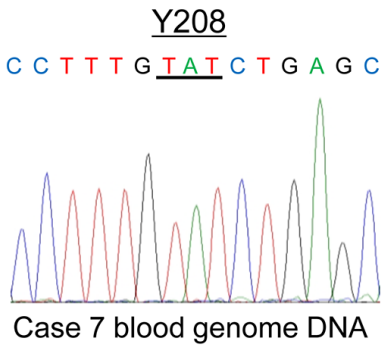

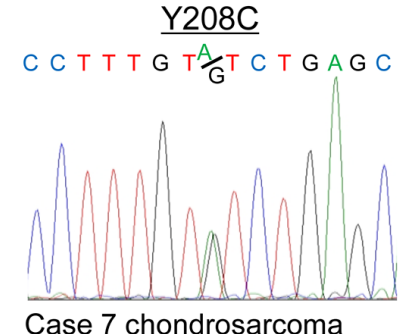

Case 7 chondrosarcoma

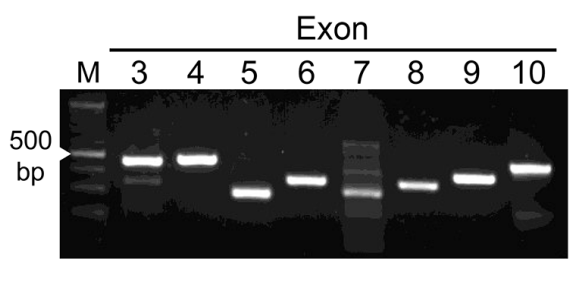

D

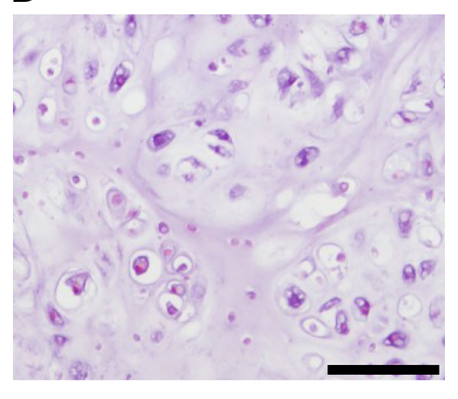

Figure 1. Detection and characterization of the canine IDH1 Y208C mutation. (A) The amino acid sequence comparison of human (NP_005887.2) and canine (BBC43078.1) IDH1. A total of 401/414 residues were identical. The bold residues represent R132 and Y208, respectively. (B) Eight exons were amplified from genomic DNA isolated from canine CS tissues (left). Electropherogram results demonstrated a single PCR band amplification (right). Amplicon sizes are provided under the left panel. (C) Electropherograms of Sanger sequencing conducted on CS cases 7 and 12. (D) Photomicrographs of canine CS in case number 7, which demonstrates representative CS pathogenesis, as indicated by hematoxylin and eosin staining (scale bar, $50 \mu \mathrm{m}$ ). IDH1, isocitrate dehydrogenase 1; CS, chondrosarcoma; Ex, exon.

$\mathrm{R} 132 \mathrm{H}$ mutant of cIDH1 was significantly lower than that in WT, and Y208C showed moderate productivity in both HeLa and MDCK cells (Fig. 2A). Production of $\alpha-\mathrm{KG}$ in HeLa cells was weakened in both $\mathrm{R} 132 \mathrm{H}$ and $\mathrm{Y} 208 \mathrm{C}$ mutant transfection compared to in WT transfection (Fig. 2B).

Overexpression of the Y208C mutant did not lead to HIF-la induction. To evaluate HIF-1 $\alpha$ induction by overexpression of the cIDH1 mutant, a reporter assay was performed to measure under the control of a promoter containing hypoxic response element sites (27). Hypoxia caused by $10 \mu \mathrm{M} \mathrm{CoCl}_{2}$ stimulation and overexpression of the R132H mutant led to the induction of HIF-1 $\alpha$ reporter activities, but the Y208C mutant did not (Fig. 2C). HIF-1 $\alpha$ protein expression was also induced by $\mathrm{CoCl}_{2}$ stimulation and R132H expression, but not Y208C (Fig. 2D).

Y208C mutation results in a conformational change in the dimerization form of IDHI. To predict the functional alteration based on the IDH1 mutation, the protein structure editing tool in the UCSF Chimera software package was used to analyze the possible structural outcomes of Y208C substitutions. Y208 is located adjacent to the binding surface of the IDH1 dimerization form. Y208 showed hydrogen bonds with amino acids belonging to the intra-strand W245, E247, R249, M254, Q257, and W267 (Fig. 3A). The Rotamers tool allows amino acid side chain rotamers to be viewed and evaluated (25). The best rotamers for $\mathrm{C} 208$ were selected based on their side-chain torsion as well as on the probability values in the rotamer library and in the context of the structural environment. These calculations revealed that the Y208C substitution disrupted or reduced the inter-strand hydrogen bond with the side chains of W245, E247, and Q257 (Fig. 3B). MTH and PD assays, which examined the binding activities between WT and mutant cIDH1, showed no significant change in WT-R132H heterodimerization and attenuated binding activity in the WT-Y208C mutant (Fig. 3C, D). The dimerization ability of cIDH1 transfected into HeLa cells was assessed using a glutaraldehyde cross-linking assay. Cell lysates from HeLa cells expressing HA-tagged WT, R132H or Y208 mutant were treated first with glutaraldehyde crosslinker and then analyzed by western blotting method. Both WT and R132H mutants formed dimers following the glutaraldehyde treatment, however, the Y208C mutant formed a weak dimer (Fig. 3E).

R132H and Y208C mutations did not affect the alteration of NOX activities. NOX activity in WT or mutant 


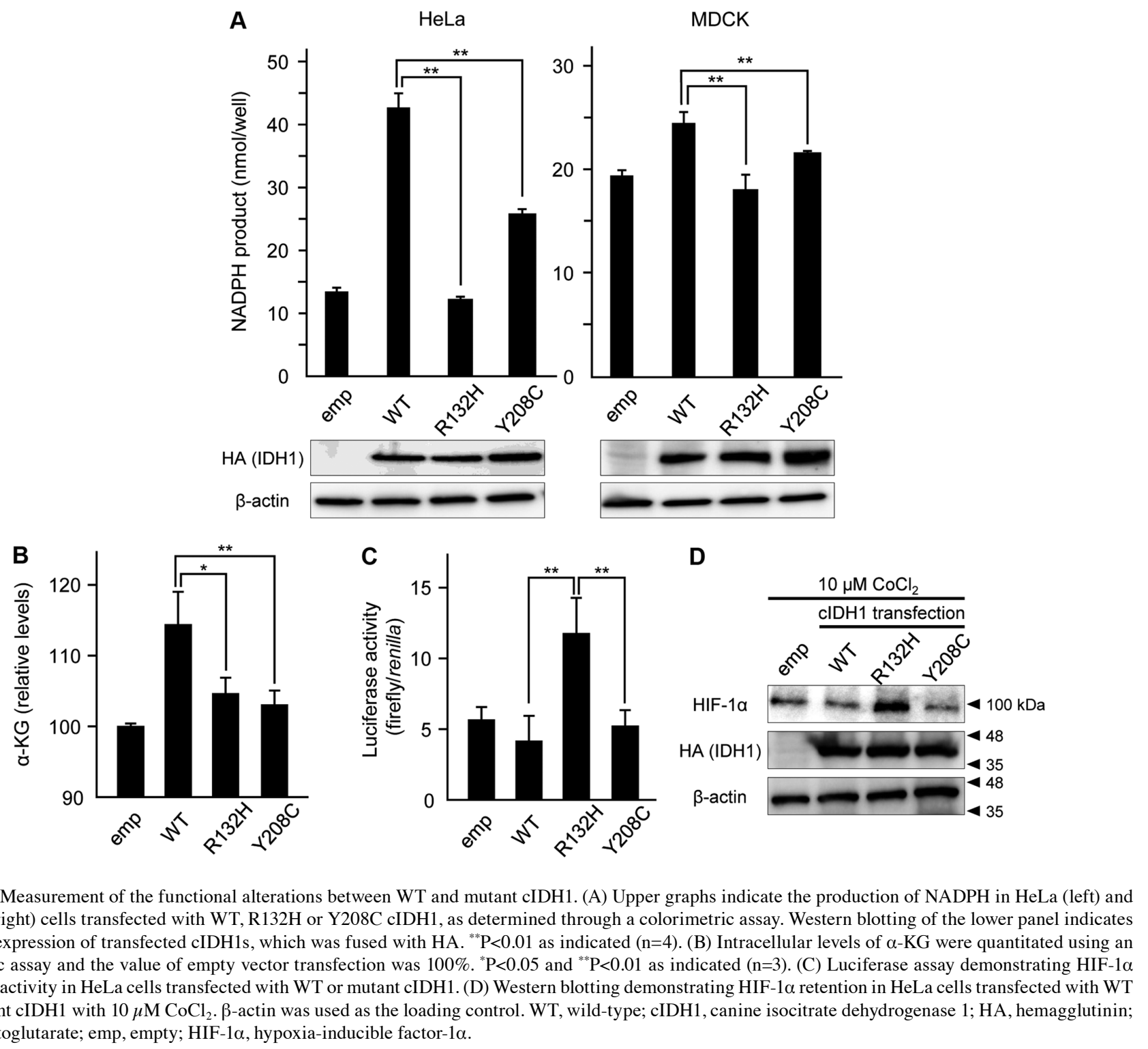

cIDH1-transfected HeLa cells was assessed. The WT of cIDH1-transfected cells showed a significant increase compared with the empty vector-transfected control, but the R132H and Y208C mutant transfectants did not show (Fig. 4).

\section{Discussion}

Two cases of novel Y208C mutations in CS tissues were not detected in the genomic DNA isolated from blood; therefore, the Y208C mutation appeared to be spontaneous somatic mutations. Although the sensitivity for detection by Sanger sequencing of R132 mutation in human cases is sometimes low (28), the Sanger sequencing peak of mutated alleles was definitive in the two cases of Y208C mutation in this study. Since there are more sensitive detection methods, such as allele-specific oligonucleotide-PCR and pyrosequencing $(29,30)$, using these methods may further increase the positive mutation rate. Deep sequencing analysis has been performed in canine glioma cases $(23,31)$, but the Y208C mutation was not detected; therefore, the Y208C mutation may be a unique mutation in CS. Two cases of
c.623A $>C$ and p.Y208C mutations in human liver cancer were archived in the Catalog of Somatic Mutations In Cancer (COSMIC; https://cancer.sanger.ac.uk/cosmic) database (32), but the gene function alterations were not described. $\mathrm{Y} 208 \mathrm{H}$ (rs587778402) and Y208C (rs186787509) mutations in human IDH1 were also studied in the NCBI SNP database (https://www.ncbi.nlm.nih.gov/snp). Histological analysis showed that there was no remarkable difference compared with other CS tissues. Because the number of cases is still small, it is possible that further histological analysis of CS with Y208C mutation in IDH1 would lead to the identification of the peculiar characteristics of the Y208C mutation. The production amount of $\alpha$-KG was also measured in the cIDH1 transfectant HeLa cell accordingly. The R132H and Y208C mutant transfectant showed significant attenuation of $\alpha-\mathrm{KG}$ production when compared with WT transfection. This data shows that the Y208C mutation of cIDH1 leads to the loss-of catalytic function from isocitrate to $\alpha-\mathrm{KG}$, and decreases the NADPH biosynthesis accordingly.

The R132H mutated IDH1 proteins lose normal catalytic activity for $\alpha-K G$ and produce less NADPH. Instead, the 
A

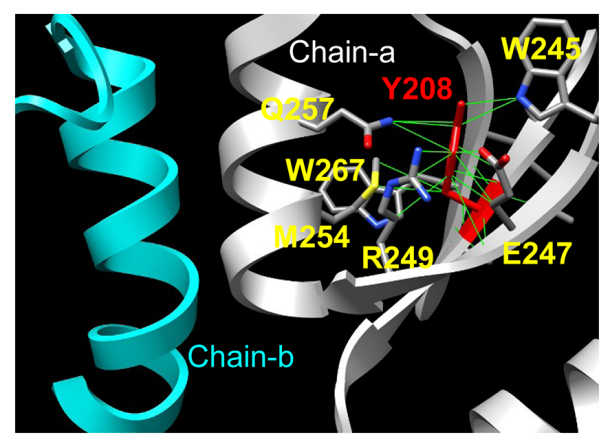

C

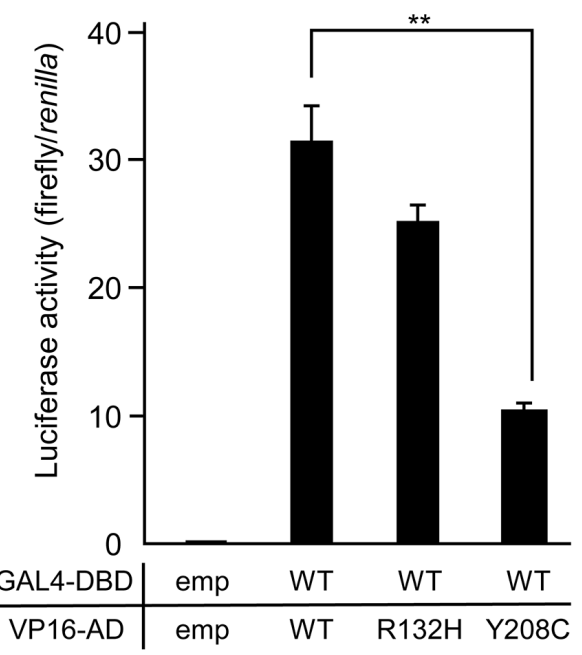

B C208 contacts

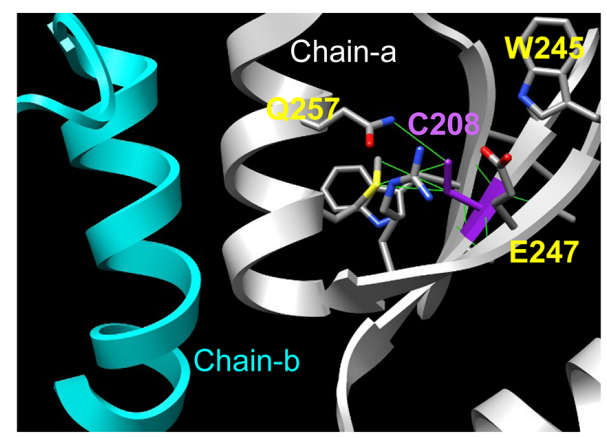

D

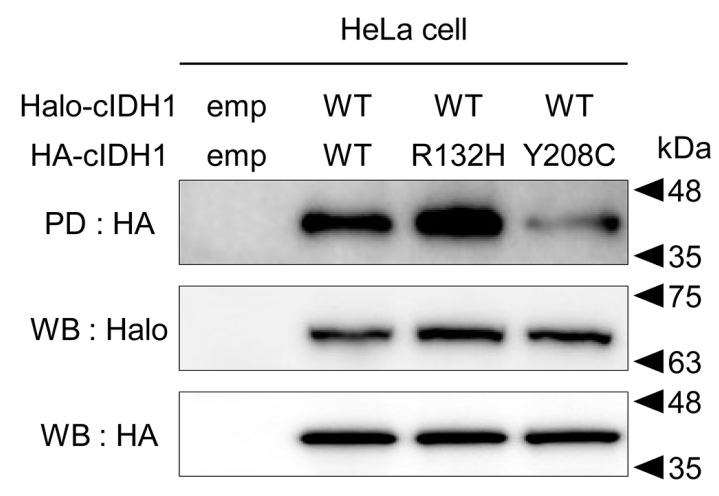

E

HeLa cell

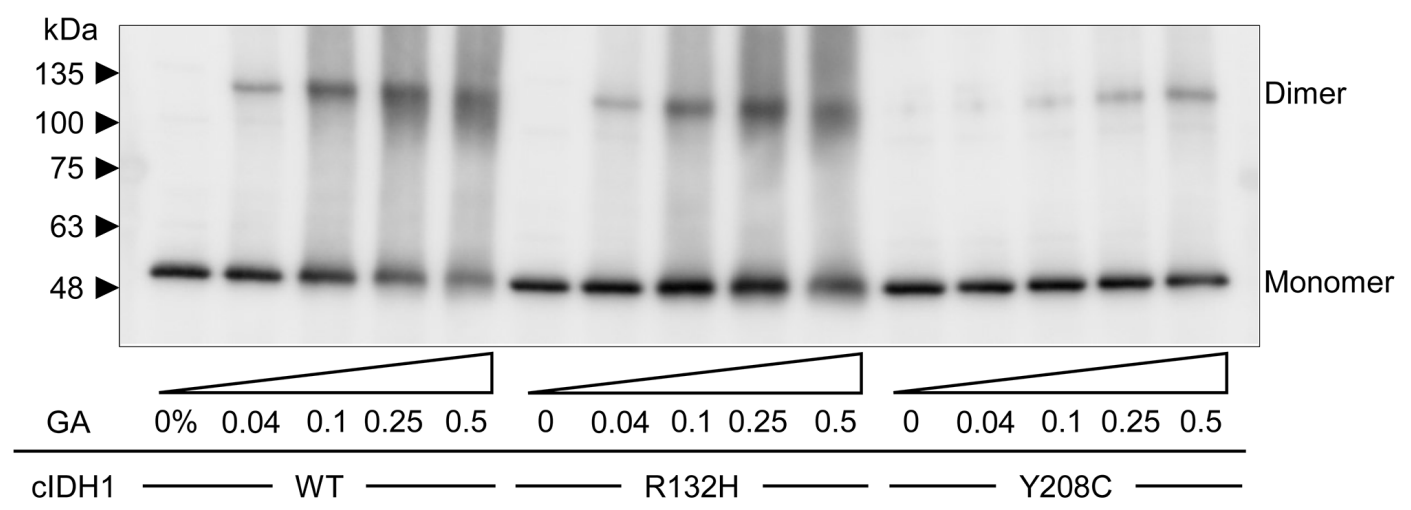

Figure 3. In silico and cell biology analysis of the effect of Y208C mutation dimerization ability. Two cIDH1 proteins are depicted as magenta (chain-a) and gray (chain-b) ribbons. The Y208 residue (red) was mutated to Cys (purple). The contacts between residues at the (A) Y208 or (B) C208 positions in the chain were calculated. The amino acid residues linking Y208 and C208 are colored gray. The solid green lines denote stable contacts, as determined using the Chimera program. (C) Binding intensities between GAL4-DBD and VP16-AD fused with the WT and cIDH1 mutants. Data are presented as the mean \pm SD $(\mathrm{n}=4)$. ${ }^{* *} \mathrm{P}<0.01$ as indicated. (D) A pull-down assay was performed for WT and mutated cIDH1 cloned into pFN21A and pMACS Kk.HA-C plasmid vectors. Halo-tagged cIDH1 and HA-tagged cIDH1 were subsequently analyzed using the indicated antibodies. (E) Glutaraldehyde cross-linking analysis of WT, R132H and Y208C cIDH1 mutants. Samples were separated by polyacrylamide gel electrophoresis and immunoblotted with an anti-HA antibody. cIDH1, canine isocitrate dehydrogenase 1; GAL4-DBD, GAL4-DNA binding domain; VP16-AD, VP16 activation domain; HA, hemagglutinin; WT, wild-type; emp, empty; WB, western blotting; PD, pull-down; GA, glutaraldehyde.

abnormal enzymatic activity produces $2-\mathrm{HG}$ and consumes NADPH (33). Therefore, we investigated the formation of NADPH in multiple types of cIDH1-overexpressing HeLa and MDCK cells using colorimetric analysis. Previous studies have used HeLa and MDCK cell lines for the analysis of the biological reaction against IDH1 overexpression $(20,14,34)$. Compared with the $\mathrm{R} 132 \mathrm{H}$ mutation transfectant, which showed a significant decrease in NADPH production, the Y208C transfectant showed moderate reduction of products in both cell types. These data indicate that the Y208C mutation diminished the isocitrate dehydrogenation activity, but not at lower levels than the R132H mutation.

The R132H mutation of IDH1 produces 2-HG from $\alpha-\mathrm{KG}$, which reduces $\alpha-\mathrm{KG}$-dependent prolyl hydroxylases, which regulate HIF-1 $\alpha$ levels (35). HeLa cells transfected with the $\mathrm{R} 132 \mathrm{H}$ mutant of cIDH1 showed an increase in HIF- $1 \alpha$ promoter activity and retention of HIF1- $\alpha$ protein, but Y208C mutant transfection did not change compared with 


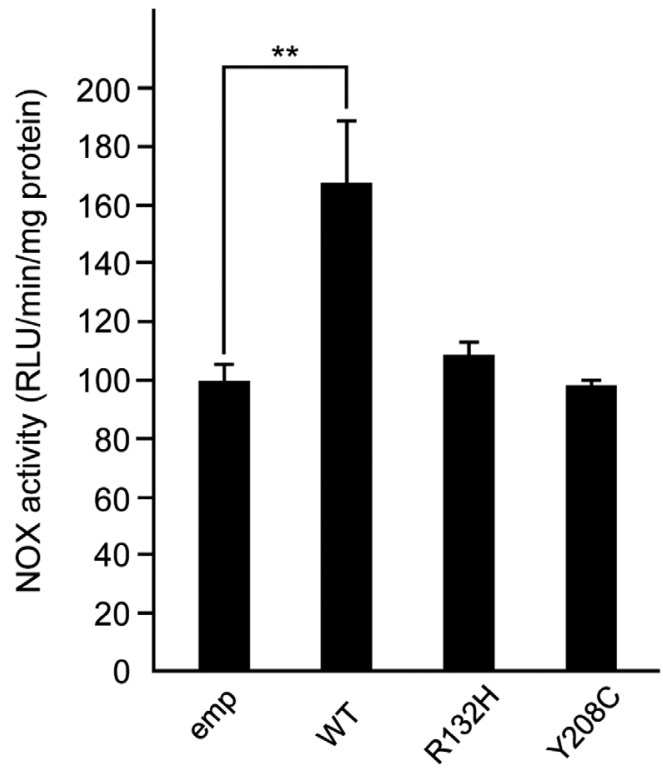

Figure 4. Generation of cellular ROS by NOX in cIDH1-transfected cells. Cellular NOX activity in WT and mutant cIDH1-transfected cells. Temporal ROS generation (RLU/mg protein) by NOX was detected using lucigenin chemiluminescence. Data are presented as the mean $\pm S D(n=3) .{ }^{* *} P<0.01$ as indicated. ROS, reactive oxygen species; NOX, NADPH oxidase; cIDH1, canine isocitrate dehydrogenase 1; WT, wild-type; emp, empty.

parental cells. These data suggest that the Y208C mutation has a different mechanism for tumorigenesis than the R132 mutation.

In Fig. 1A, as seen clearly, the amino acids sequence of human and canine IDH1 are highly conserved; hence, we thought that the protein structure data of human IDH1 (PDB ID: 5YFM) could be extrapolated to analyze the Y208C mutation in cIDH1. Our in silico simulation of amino acid substitution from tyrosine to cysteine showed the loss of contact against intra-strand amino acids located in an $\alpha$-helix structure (amino acid residues 251 to 261 of GenBank accession no. BBC43078.1), which forms the binding surface of the homodimer (36), because the side chain of cysteine was shorter than that of the tyrosine side chain. As predicted by in silico analysis, Y208C mutation attenuated the binding ability against WT of IDH1 by MTH, PD assay and protein cross-linked electrophoresis, however not for the WT-R132H interaction. Both $\mathrm{R} 132 \mathrm{H}$ and $\mathrm{Y} 208 \mathrm{C}$ mutants attenuate isocitrate dehydrogenase activity. The $\mathrm{R} 132 \mathrm{H}$ mutant produces carcinogenic $2-\mathrm{HG}$ from $\alpha-\mathrm{KG}$, which causes a reduction in the NADPH production. On the other hand, the Y208C mutant cannot form dimers, which causes attenuation of enzymatic activity. These phenomena suggest that the carcinogenic mechanisms are different between R132H and Y208C mutations in IDH1.

IDH1 mutation causes a change in NADPH production, so we predicted that IDH1 mutation may affect NOX activity, which reflects the amount of reactive oxygen species (ROS). To elucidate the cause of tumorigenesis due to the Y208C mutation, we analyzed NOX activity in cIDH1-transfected cells. NOX activity was significantly higher in WT IDH1-transfected cells, and there was no significant difference between the negative control and both R132H and Y208C mutant transfected cells. This result suggests that WT IDH1 could produce more
NADPH, a source of ROS, but IDH1 mutants lose the ability to produce more NADPH; therefore, mutant transfected cells could not produce ROS. Since there was no difference in the results of ROS productivity between R132H and Y208C, the mechanism of carcinogenesis of the Y208C mutation remains unclear. This study is the first experimental report to describe the relationship between canine IDH1 mutation and NOX activity. Future studies will need to elucidate the mechanism of tumorigenesis of the Y208C mutation. Furthermore, future studies will have to look for Y208C mutations in various tumors.

In conclusion, we identified for the first time Y208C spontaneous somatic mutations of canine IDH1 in chondrosarcomas and assessed the impact of these mutations on IDH1 functions. Y208C mutation attenuated the NADPH production ability but did not enhance HIF- $1 \alpha$ retention in $\mathrm{CoCl}_{2}$-treated cells. This phenomenon was caused by the attenuation of the dimerization ability of the Y208C mutation. We hope that the precise analysis of IDH1 functional changes can help elucidate the tumorigenesis involvement of the Y208C IDH1 mutation.

\section{Acknowledgements}

Not applicable.

\section{Funding}

This work was supported by KAKENHI scientific research grants from the Ministry of Education, Culture, Sports, Science and Technology of Japan (grant nos. 18H02334 and 19K06390).

\section{Availability of data and materials}

The datasets used and/or analyzed during the current study are available from the corresponding author on reasonable request.

\section{Authors' contributions}

SK, MS, MMi, MMo, KI, MW and KO designed the study and performed the bioinformatics analysis. SK, MU, NK, MMa, YM, DA, ASE, EO and TO performed the laboratory experiments. KO and YT performed statistical analysis and wrote the manuscript. MW, YT, TO and KO supervised the study. All authors have read and approved the final version of the manuscript.

\section{Ethics approval and consent to participate}

The current study was approved by the University Ethics Committee of the Department of Veterinary Pathology, School of Veterinary Science, Nippon Veterinary and Life Science University (approval no. 11-50, 27 May 2018).

\section{Patient consent for publication}

Not applicable.

\section{Competing interests}

The authors declare that they have no competing interests. 


\section{References}

1. Sahm F, Reuss D, Koelsche C, Capper D, Schittenhelm J, Heim S, Jones DT, Pfister SM, Herold-Mende C, Wick W, et al: Farewell to oligoastrocytoma: In situ molecular genetics favor classification as either oligodendroglioma or astrocytoma. Acta Neuropathol 128: 551-559, 2014.

2. Lapointe S, Perry A and Butowski NA: Primary brain tumours in adults. Lancet 392: 432-446, 2018.

3. Parsons DW, Jones S, Zhang X, Lin JC, Leary RJ, Angenendt P, Mankoo P, Carter H, Siu IM, Gallia GL, et al: An integrated genomic analysis of human glioblastoma multiforme. Science 321: 1807-1812, 2008.

4. Reitman ZJ and Yan H: Isocitrate dehydrogenase 1 and 2 mutations in cancer: Alterations at a crossroads of cellular metabolism. J Natl Cancer Inst 102: 932-941, 2010.

5. Bleeker FE, Lamba S, Rodolfo M, Scarpa A, Leenstra S, Vandertop WP and Bardelli A: Mutational profiling of cancer candidate genes in glioblastoma, melanoma and pancreatic carcinoma reveals a snapshot of their genomic landscapes. Hum Mutat 30: E451-E459, 2009.

6. Yan H, Parsons DW, Jin G, McLendon R, Rasheed BA, Yuan W, Kos I, Batinic-Haberle I, Jones S, Riggins GJ, et al: IDH1 and IDH2 mutations in gliomas. N Engl J Med 360: 765-773, 2009.

7. MardisER,Ding L, Dooling DJ,Larson DE, McLellan MD,Chen K, Koboldt DC, Fulton RS, Delehaunty KD, McGrath SD, et al: Recurring mutations found by sequencing an acute myeloid leukemia genome. N Engl J Med 361: 1058-1066, 2009.

8. Lugowska I, Teterycz P, Mikula M, Kulecka M, Kluska A, Balabas A, Piatkowska M, Wagrodzki M, Pienkowski A, Rutkowski P and Ostrowski J: IDH1/2 mutations predict shorter survival in chondrosarcoma. J Cancer 9: 998-1005, 2018.

9. Waitkus MS, Diplas BH and Yan H: Biological role and therapeutic potential of IDH mutations in cancer. Cancer Cell 34: 186-195, 2018.

10. Dang L, White DW, Gross S, Bennett BD, Bittinger MA, Driggers EM, Fantin VR, Jang HG, Jin S, Keenan MC, et al: Cancer-associated IDH1 mutations produce 2-hydroxyglutarate. Nature 462: 739-744, 2009.

11. Gross S, Cairns RA, Minden MD, Driggers EM, Bittinger MA, Jang HG, Sasaki M, Jin S, Schenkein DP, Su SM, et al: Cancer-associated metabolite 2-hydroxyglutarate accumulates in acute myelogenous leukemia with isocitrate dehydrogenase 1 and 2 mutations. J Exp Med 207: 339-344, 2010.

12. Schnittger S, Haferlach C, Ulke M, Alpermann T, Kern W and Haferlach T: IDH1 mutations are detected in 6.6\% of 1414 AML patients and are associated with intermediate risk karyotype and unfavorable prognosis in adults younger than 60 years and unmutated NPM1 status. Blood 116: 5486-5496, 2010.

13. Wajner M, Latini A, Wyse AT and Dutra-Filho CS: The role of oxidative damage in the neuropathology of organic acidurias: insights from animal studies. J Inherit Metab Dis 27: 427-448, 2004.

14. Zhao S, Lin $\mathrm{Y}, \mathrm{Xu}$ W, Jiang W, Zha Z, Wang $\mathrm{P}$, Yu W, Li Z, Gong L, Peng Y, et al: Glioma-derived mutations in IDH1 dominantly inhibit IDH1 catalytic activity and induce HIF-1alpha. Science 324: 261-265, 2009.

15. Calvert AE, Chalastanis A, Wu Y, Hurley LA, Kouri FM, Bi Y, Kachman M, May JL, Bartom E, Hua Y, et al: Cancer-associated IDH1 promotes growth and resistance to targeted therapies in the absence of mutation. Cell Rep 19: 1858-1873, 2017.

16. Wahl DR, Dresser J, Wilder-Romans K, Parsels JD, Zhao SG, Davis M, Zhao L, Kachman M, Wernisch S, Burant CF, et al: Glioblastoma therapy can be augmented by targeting IDH1-mediated NADPH biosynthesis. Cancer Res 77: 960-970, 2017.

17. Zarei M,Lal S, Parker SJ, Nevler A, Vaziri-Gohar A, Dukleska K, Mambelli-Lisboa NC, Moffat C, Blanco FF, Chand SN, et al: Posttranscriptional upregulation of IDH1 by HuR establishes a powerful survival phenotype in pancreatic cancer cells. Cancer Res 77: 4460-4471, 2017.

18. Dobson JM, Samuel S, Milstein H, Rogers K and Wood JL: Canine neoplasia in the UK: Estimates of incidence rates from a population of insured dogs. J Small Anim Pract 43: 240-246, 2002.
19. Reitman ZJ, Olby NJ, Mariani CL, Thomas R, Breen M, Bigner DD, McLendon RE and Yan H: IDH1 and IDH2 hotspot mutations are not found in canine glioma. Int J Cancer 127: 245-246, 2010.

20. Kawakami S, Ochiai K, Azakami D, Kato Y, Michishita M, Morimatsu M, Ishiguro-Oonuma T, Onozawa E, Watanabe M and Omi T: R132 mutations in canine isocitrate dehydrogenase 1 (IDH1) lead to functional changes. Vet Res Commun 42: 49-56, 2018.

21. Kaneko MK, Tian W, Takano S, Suzuki H, Sawa Y, Hozumi Y, Goto K, Yamazaki K, Kitanaka C and Kato Y: Establishment of a novel monoclonal antibody SMab-1 specific for IDH1-R132S mutation. Biochem Biophys Res Commun 406: 608-613, 2011.

22. Kato Y: Specific monoclonal antibodies against IDH1/2 mutations as diagnostic tools for gliomas. Brain Tumor Pathol 32: 3-11, 2015.

23. Amin SB, Anderson KJ, Boudreau CE, Martinez-Ledesma E, Kocakavuk E, Johnson KC, Barthel FP, Varn FS, Kassab C, Ling X, et al: Comparative molecular life history of spontaneous canine and human gliomas. Cancer Cell 37: 243-257.e7, 2020.

24. Misdorp W, Else RW, Hellmen E and Lipscomb TP: Histological classification of mammary tumors of the dog and the cat. In: World Health Organization International Histological Classification of Tumors of Domestic Animals. Schulman FY (ed). Vol 7. 2nd Series. Armed Forces Institute of Pathology, Washington, DC, p58, 1999.

25. Pettersen EF, Goddard TD, Huang CC, Couch GS, Greenblatt DM, Meng EC and Ferrin TE: UCSF Chimera-a visualization system for exploratory research and analysis. J Comput Chem 25: 1605-1612, 2004.

26. Jalil JE, Perez A, Ocaranza MP, Bargetto J, Galaz A and Lavandero S: Increased aortic NADPH oxidase activity in rats with genetically high angiotensin-converting enzyme levels. Hypertension 46: 1362-1367, 2005.

27. Emerling BM, Platanias LC, Black E, Nebreda AR, Davis RJ and Chandel NS: Mitochondrial reactive oxygen species activation of p38 mitogen-activated protein kinase is required for hypoxia signaling. Mol Cell Biol 25: 4853-4862, 2005.

28. Preusser M, Wohrer A, Stary S, Hoftberger R, Streubel B and Hainfellner JA: Value and limitations of immunohistochemistry and gene sequencing for detection of the IDH1-R132H mutation in diffuse glioma biopsy specimens. J Neuropathol Exp Neurol 70: 715-723, 2011.

29. Ashraf S, Noguera NI, Di Giandomenico J, Zaza S, Hasan SK and Lo-Coco F: Rapid detection of IDH2 (R140Q and R172K) mutations in acute myeloid leukemia. Ann Hematol 92: 1319-1323, 2013.

30. Catteau A, Girardi H, Monville F, Poggionovo C, Carpentier S, Frayssinet V, Voss J,Jenkins R Boisselier B, Mokhtari K, et al: A new sensitive PCR assay for one-step detection of $12 \mathrm{IDH} 1 / 2$ mutations in glioma. Acta Neuropathol Commun 2: 58, 2014.

31. Truve K, Dickinson P, Xiong A, York D, Jayashankar K, Pielberg G, Koltookian M, Muren E, Fuxelius HH, Weishaupt $\mathrm{H}$, et al: Utilizing the dog genome in the search for novel candidate genes involved in glioma development-genome wide association mapping followed by targeted massive parallel sequencing identifies a strongly associated locus. PLoS Genet 12: e1006000, 2016.

32. Tate JG, Bamford S, Jubb HC, Sondka Z, Beare DM, Bindal N, Boutselakis H, Cole CG, Creatore C, Dawson E, et al: COSMIC: The catalogue of somatic mutations in cancer. Nucleic Acids Res 47: D941-D947, 2019.

33. Huang LE: Friend or foe-IDH1 mutations in glioma 10 years on. Carcinogenesis 40: 1299-1307, 2019.

34. Koyasu S, Shimizu Y, Morinibu A, Saga T, Nakamoto Y, Togashi $\mathrm{K}$ and Harada $\mathrm{H}$ : Increased ${ }^{14} \mathrm{C}$-acetate accumulation in IDH-mutated human glioblastoma: Implications for detecting IDH-mutated glioblastoma with ${ }^{11} \mathrm{C}$-acetate PET imaging. J Neuro Oncol 145: 441-447, 2019.

35. Zhao S and Guan KL: IDH1 mutant structures reveal a mechanism of dominant inhibition. Cell Res 20: 1279-1281, 2010.

36. Vinekar R, Verma $C$ and Ghosh I: Functional relevance of dynamic properties of Dimeric NADP-dependent isocitrate dehydrogenases. BMC Bioinformatics 13 (Suppl 17): S2, 2012.

c) (i) $(-)$ This work is licensed under a Creative Commons Attribution-NonCommercial-NoDerivatives 4.0 International (CC BY-NC-ND 4.0) License. 